\title{
Forced Nonradial Oscillations in Early-Type Rotating Stars
}

\author{
M. G. Witte, G. J. Savonije
}

Astronomical Institute "Anton Pannekoek", University of Amsterdam, Kruislaan 403, NL-1098 SJ, Amsterdam

A method of calculating nonradial oscillations in rotating stars is presented. Using this method, we are able to calculate the spectrum of $\mathrm{g}$-, $\mathrm{f}$ - and $\mathrm{p}$-mode eigenfunctions of a star for different stellar rotation speeds, and also the spectrum of rotational $\mathbf{r}$ modes. Stability of the modes as a function of stellar rotation speed can be investigated. By regarding the response of a star which undergoes periodic deformations due to the gravitational force of an orbiting companion as a forced nonradial oscillation, the problem of determining the eigenfrequencies of the star becomes one of finding resonances with the forcing potential. Expanding the potential of the orbiting (point mass) companion in terms of the usual spherical functions, the response of the star to each tidal term $\Phi_{l m}=-f_{l m} r^{l} P_{l}^{m}(\cos \vartheta) \cos (\sigma t-m \varphi)$, with $l$ and $m$ fixed, can be calculated separately. By varying the forcing frequency $\sigma$ we are then able to calculate the stellar spectrum. To calculate the response of the star we numerically solve the fully nonadiabatic, but linearised hydrodynamical equations for the star, in which the Coriolis forces due to stellar rotation are fully taken into account. To this end we utilise an implicit 2D finite difference scheme which solves the equations on an $(r, \vartheta)$ grid. A calculated solution describes the steady state in which the power $\sigma \mathcal{T}$ due to the external driving force is in equilibrium with the internal damping. For results and more references see Witte \& Savonije (1999).

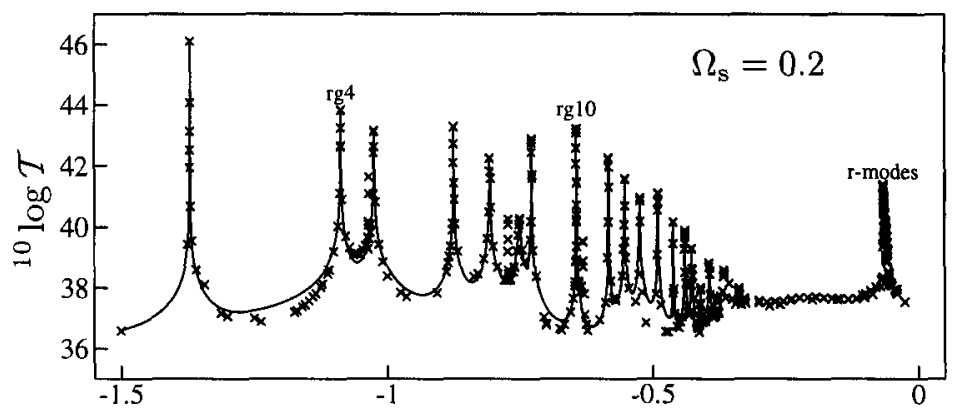

Figure 1. Oscillation spectrum of a $10 M_{\odot}$ rotating star with $X_{\mathrm{c}}=0.4$.

\section{Reference}

Witte, M. G. \& Savonije, G. J. 1999, A\&A, 341, 842 